\title{
Integral Collocation Approximation Methods for the Numerical Solution of Linear Integro Differential Equations.
}

\author{
${ }^{1}$ Taiwo, O. A and ${ }^{2}$ Abubakar, A \\ ${ }^{I}$ Department of Mathematics, University of Ilorin, Nigeria. \\ ${ }^{2}$ Department of Mathematics and Computer Science, Ibrahim Badamasi Babangida University, Lapai, Nigeria.
}

\begin{abstract}
In this paper, we employed Standard and Perturbed Integral Collocation methods to find numerical solution of ordinary differential equations. Power series form of approximation is used as basis function and Chebyshev Polynomial is used as perturbation term in the case of perturbed Integral Collocation Method. Numerical Computations are carried out to illustrate the application of the methods and also the results obtained by the methods are compared in terms of accuracy and computations involved in the two methods. Two examples each of first and second orders linear integro differential equations are solved to demonstrate the methods.
\end{abstract}

\section{Introduction}

Integro differential equation (IDE) is an important branch of modern mathematics and a arise frequently in many applied areas which include engineering, mechanics, physics ,astronomy, biology ,economics, potential theory and electrostatic[2].

This paper concerns the development of integral collocation approximation methods by power series as our basis function for the solution of first order integro differential equations .

Many different method have been used to obtain the solution of linear and nonlinear integro differential equations ,such methods include the Successive Approximation Method, Homotopy Perturbation Method [6], Adomian Decomposition Method (ADM [13], Wavelet Methods (See [4],[5] ) and so on , to mention just a few . [1] used the integrated formulation of the Tau method and Error Estimation for over determined differential equations which actually motivated the beginning of this work.

For the purpose of our discussion, we consider the general nth order integro differential equation of the form :

$$
L y(x) \cong \sum_{i=0}^{n}\left(p_{i} x^{i} \frac{d^{i}}{d x^{i}}\right) y(x)+\int_{a}^{b} k(x, t) y(t) d t=f(x)
$$

Subject to the conditions

$$
\operatorname{Ly}(x) \quad \cong \sum_{i=0}^{N} a_{i} y^{i}\left(x_{i}\right)=\alpha_{k}
$$

Where $p_{i}$, are given real numbers, $x_{i}$ are point belonging to the internal $a \leq x \leq b$ at which the conditions are satisfied, $f(x)$ can be polynomial or transcendental or hyperbolic functions and or $(\gamma \geq o)$ are constant to be determined.

Here, we let

$$
\iiint \ldots k \ldots \int g(x) d x
$$

Denote the indefinite integration applied to the $g(x) k$ times and denote $I_{L}=\iiint \ldots . . n \ldots . . \int L() d$.

\section{Method1. Standard Integral Collocation Method}

\section{Numerical solution Techniques}

This method used the idea of [1] as applied to solve non over determined differential equations.

In [1], $f(x)$ in (1) is a polynomial of degree less or equal to the order of or the differential equation considered .these methods handle non polynomial or transcendental function which is an added advantage over [1] (See[11], [12]).

The method does not require truncation or approximation of non polynomial functions.

Also the problem of over determination does not arise in these methods. Without loss of generality, we integrated both sides of (1), we have 


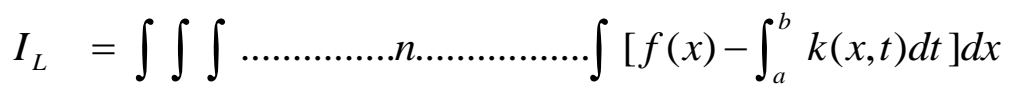

This implies,

$$
\iiint \ldots \ldots \ldots . . . \ldots \ldots . . \int \sum_{i=o}^{n}\left(p_{i} x^{i} \frac{d^{i}}{d x^{i}}\right) d x=\iiint \ldots \ldots \ldots . . \ldots \ldots . \int\left[f(x)-\int_{a}^{b} k(n, t) y(t) d t\right] d x
$$

We assumed an approximation solution of the form

$$
y(x)=y_{N}(x)=\sum_{r=o}^{N} a_{r} x^{i}
$$

Thus, (6) is substituted into (5), we have

Hence, we collocate (7) at point to have

$$
\begin{aligned}
& \iiint \ldots \ldots . . . \ldots \ldots . . . \int\left(\sum_{i=o}^{n} p_{i} x^{i} \frac{d^{i}}{d x^{i}} y_{N}\left(x_{K}\right) d x_{K}\right. \\
& =\iiint \ldots \ldots . . \ldots \ldots . \int\left[f\left(x_{K}\right)-\int_{a}^{b} k\left(x_{K}, t\right) y_{N}(t) d t\right] d x_{K}
\end{aligned}
$$

Where,

$$
x_{K}=a+\frac{(b+a) k}{N+1} ; \quad k=1,2 \ldots \ldots \ldots \ldots \ldots . . n+1
$$

Thus (8) gives $(N+1)$ algebraic linear system of equations in $(N+1)$ unknown constants $a_{r}(r \geq o)$. These $(N+1)$ algebraic linear equations are then solved by Gaussian Elimination method to obtain the unknown constants $a_{r} \quad(r \geq o)$ which are then substituted back into (6) to obtain the approximate solution for the value of $\mathrm{N}$.

\section{Method 2: $\quad$ Perturbed Integral Collocation Method.}

The perturbed integral collocation method is an attempt to improve the accuracy and efficiency of the standard integral collocation method .

In order to apply this method, we employed the ideas of [11, 12] and the approximation solution (6) is substituted into a slightly perturbed (7) to get

$$
\begin{aligned}
& \iiint \ldots \ldots . . \ldots \ldots . . \int \sum_{i=o}^{n}\left(p_{i} x^{i} \frac{d^{i}}{d x^{i}}\right) y_{N}(x) d x \\
& =\iiint \ldots . . n \ldots \ldots . . \int\left[(x)-\int_{a}^{b} k\left(x_{1} t\right) y_{N}(t) d t\right] d x+H_{n}(x)
\end{aligned}
$$

Together with the conditions

$$
L^{*} y_{N}\left(x_{K}\right)=\alpha_{K} ; k=(1) n
$$

Where $H_{n}(x)=\sum_{r=o}^{n} \tau_{r} T_{r}(x)$

And $T_{r}(x)$ are the Chebyshev Polynomials valid in $a \leq x \leq b$ and defined by

$$
T_{r}(x)=\cos \left[n \cos ^{-1}\left(\frac{2 x-a-b}{b-a}\right)\right] ; \quad a \leq x \leq b
$$

Substituting (12) into (10), we have

$$
\begin{aligned}
& \iiint \ldots \ldots . . \ldots \ldots . . \int \sum_{i=o}^{n}\left(p_{i} x^{i} \frac{d^{i}}{d x^{i}}\right) y_{N}(x) d x \\
& \iiint \ldots \ldots . . \ldots \ldots . . \int \sum_{i=o}^{n}\left(p_{i} x^{i} \frac{d^{i}}{d x_{k}^{i}}\right) y_{N}(x) d x
\end{aligned}
$$


$=\iiint \ldots \ldots . . \ldots \ldots . . \int\left[f(x)-\int_{a}^{b} k(x, t) y_{N}(t) d t\right] d x+\tau_{1} T_{1}(x)+\ldots \ldots . .+\tau_{N} T_{N}(x)$

Thus, (14) is collocated at the point $x=x_{K}$; we have

$$
\begin{aligned}
& \iiint \ldots \ldots . . \ldots \ldots . . \int \sum_{i=o}^{n}\left(p_{i} x_{k}{ }^{i} \frac{d^{i}}{d x^{i}}\right) y_{N}\left(x_{k}\right) d x \\
& \iiint \ldots \ldots . \ldots \ldots . . \int \sum_{i=o}^{n}\left(p_{i} x_{k}^{i} \frac{d^{i}}{d x_{k}^{i}}\right) y_{N}\left(x_{k}\right) d x \\
& =\iiint \ldots \ldots . . \ldots \ldots . \int\left[f\left(x_{k}\right)-\int_{a}^{b} k\left(x_{k}, t\right) y_{N}(t) d t\right] d x+\tau_{1} T_{1}\left(x_{k}\right)+\ldots \ldots .+\tau_{N} T_{N}\left(x_{k}\right)
\end{aligned}
$$

Where,

$$
x_{K}=\quad a+\frac{(b-a) k}{N+n+1} \quad ; k=1,2, \ldots \ldots \ldots . . . N+n+1
$$

Hence, we have $(N+n+1)$ algebraic system of linear equations in $(N+n+1)$ unknown constants $\left(a_{r}(r \geq o), \tau_{1}, \tau_{2, \ldots, \ldots \ldots \ldots,} \tau_{N}\right)$. The linear equations are then solved by Gaussian Elimination method to obtain $(N+n=1)$ unknown constants which are then substituted back into (6) to obtain the approximate solution for value of $\mathrm{N}$.

\section{Demonstration with first order integro differentiation equation}

We consider here case $n=1$ in equation (1). Thus, we have

$$
p_{0} y(x)+p_{1} x y^{\prime}(x)+\int_{a}^{b} k(x, t) y(t) d t=f(x)
$$

We integrated the terms in (17) to have

$$
\begin{gathered}
\int_{o}^{x} p_{o} y(t) d t+\int_{o}^{x} p_{1} t y^{\prime}(t) d t+\int_{o}^{x} \int_{a}^{b} k(z, t) y(t) d t d z=\int_{o}^{x} f(z) d z \\
p_{o} \int_{o}^{x} y(t) d t+p_{1} \int_{o}^{x} t y(t) d t+\int_{o}^{x} \int_{o}^{b} k(z, t) y(t) d t d z=\int_{o}^{x} f(z) d z
\end{gathered}
$$

Hence, evaluated the integrals in (18), we have

This implies,

$$
p_{o} \int_{0}^{x} y(t) d t+p_{1}\left[[t y(t)]_{o}^{x}-\int_{o}^{x} y(t) d t\right]+\int_{o}^{x} \int_{a}^{b} k(z, t) y(t) d t d z=\int_{o}^{x} f(z) d z
$$

$$
p_{o} \int_{o}^{x} y(t) d t+p_{1}\left[x y(x)-\int_{o}^{x} y(t) d t\right]+\int_{o}^{x} \int_{o}^{b} k(z, t) y(t) d t d z=\int_{o}^{x} f(z) d z
$$

Substituting the approximation solution (6) into (19), we have

$$
p_{o} \int_{o}^{x} \sum_{r=o}^{N} a_{r} t^{r} d t+p_{1}\left[x \sum_{r=o}^{N} a_{r} x^{r}-\int_{o}^{x} \sum_{r=o}^{N} a_{r} t^{r} d t\right]+\int_{o}^{x} \int_{a}^{b} k(z, t) \sum_{r=o}^{N} a_{r} t^{r} d t d z=\int_{o}^{x} f(z) d z
$$

This implies,

$$
p_{o} \sum_{r=o}^{N} a_{r} \frac{x^{r+1}}{r+1}+p_{1}\left[\sum_{r=o}^{N} a_{r} x^{r+1}-\sum_{r=o}^{N} a_{r} \frac{x^{r+1}}{r+1}\right]+\int_{o}^{x} \int_{o}^{b} k(z, t) \sum_{r=o}^{N} a_{r} t^{r} d t d z=\int_{o}^{x} f(z) d z
$$

Further simplification gives

$$
\sum_{r=o}^{N}\left(p_{o}+r p_{1}\right) a_{r} \frac{x^{r+1}}{r+1}+\int_{o}^{x} \int_{a}^{b} k(z, t) \sum_{r=o}^{N} a_{r} t^{r} d t d z=\int_{o}^{x} f(z) d z
$$

This implies

$$
\sum_{r=o}^{N}\left(p_{o}+r p_{1}\right) a_{r} \frac{x^{r+1}}{r+1}+G(a, x)=F(x)
$$

Where, 
$G(a, x)=\int_{o}^{x} \int_{a}^{b} k(z, t) \sum_{r=o}^{N} a_{r} t^{r} d t d z$

And

$F(x)=\int_{0}^{x} f(z) d z$

Thus, from (20), we have.

$\left(p_{o}+4 p_{1}\right) a_{4} \frac{x^{5}}{5}+\ldots \ldots \ldots+\left(p_{o}+N p_{1}\right) a_{N} \frac{x^{N+1}}{N+1}+G(a, x)=f(x)$

Hence, we collocated (21) at the point $x=x_{K}$, to have

$p_{o} a_{o} x_{K}+\left(p_{o}+p_{1}\right) a_{1} \frac{x_{k}^{2}}{2}+\left(p_{o}+2 p_{1}\right) a_{2} \frac{x_{k}^{3}}{3}+\left(p_{o}+3 p_{1}\right) a_{3} \frac{x_{k}^{4}}{4}+$
$\left(p_{o}+4 p_{1}\right) a_{4} \frac{x_{k}^{5}}{5}+\ldots \ldots . . .+\left(p_{o}+N p_{1}\right) a_{N} \frac{x_{k}^{N+1}}{N+1}+G\left(a, x_{k}\right)=F\left(x_{k}\right)$,

Where,

$$
x_{i}=a+\frac{(b-a) k}{N+1}, \quad k=1.2, \quad, N+1
$$

Thus (22) is put in matrix form as

$A x=b$

Where,

$$
A=\left[\begin{array}{cccccc}
A_{11} & A_{12} & A_{13} & . \ldots \ldots . . & A_{1 N} \\
A_{21} & A_{22} & A_{23} & \ldots \ldots \ldots \ldots & A_{2 N} \\
A_{31} & A_{32} & \mathrm{~A}_{33} & \ldots \ldots \ldots \ldots & \mathrm{A}_{3 \mathrm{~N}} \\
\cdot & \cdot & \cdot & . & . \\
\cdot & \cdot & \cdot & . & & \cdot \\
A_{N 1} & A_{N 2} & A_{N 3} & \ldots \ldots \ldots & A_{N N}
\end{array}\right], x=\left[\begin{array}{c}
a_{o} \\
a_{1} \\
a_{2} \\
a_{N}
\end{array}\right] \text { and } b=\left[\begin{array}{l}
F\left(x_{1}\right) \\
F\left(x_{2}\right) \\
F\left(x_{3}\right) \\
. \\
. \\
F\left(x_{N+1}\right)
\end{array}\right]
$$

Where,

$$
\begin{aligned}
& A_{11}=p_{0} x_{1}+G\left(x_{1}\right), \backslash \\
& A_{12}=\left(p_{0}+p_{1}\right) \frac{x_{1}^{2}}{2}+G\left(x_{1}\right) \\
& A_{13}=\left(P_{O}+2 P_{1}\right) \frac{x_{1}^{3}}{3}+G\left(x_{1}\right) \\
& : \\
& A_{1 N}=\left(p_{o}+N p_{1}\right) \frac{x_{1}^{N+1}}{N+1}+G\left(x_{1}\right) \\
& A_{21}=p_{o} x_{2}+G\left(x_{2}\right) \\
& A_{22}=\left(p_{o}+p_{1}\right) \frac{x_{2}{ }^{2}}{2}+G\left(x_{2}\right)
\end{aligned}
$$




$$
\begin{aligned}
& A_{23}=\left(p_{o}+2 p_{1}\right) \frac{x_{2}{ }^{3}}{3}+G\left(x_{2}\right) \\
& : \\
& A_{2 N}+\left(p_{o}+N p_{1}\right) \frac{x_{2}{ }^{N+1}}{N+1}+G\left(x_{2}\right) \\
& A_{31}=p_{o} x_{3}+G\left(x_{3}\right) A_{32}=\left(p_{o}+p_{1}\right) \frac{x_{3}^{2}}{2}+G\left(x_{3}\right) \\
& A_{33}=\left(p_{o}+2 p_{1}\right) \frac{x_{3}^{3}}{3} G\left(x_{3}\right) \\
& : \\
& A_{3 \mathrm{~N}}=\left(p_{o}+N P_{1}\right) \frac{x_{3}{ }^{N+1}}{\mathrm{~N}+1}+G\left(x_{3}\right) \\
& : \\
& : \\
& : \\
& A_{N 1}=p_{o} x_{N}+G\left(x_{N}\right) \\
& : \\
& : \\
& A_{N N}=\left(p_{o}+p_{1}\right) \frac{x_{N}{ }^{2}}{2} G\left(x_{N}\right) \\
& :
\end{aligned}
$$

Remarks, The matrix is solved by Gaussian Elimination method to obtain the unknown constants $a_{i}(i \geq o)$ which are then substituted into the approximate solution (6)

\section{Demonstration of method for case $n=2$}

$$
p_{o} y(x)+p_{1} x y^{\prime}(x)+p_{2} x^{2} y^{\prime \prime}(x)+\int_{a}^{b} k(x, t) y(t) d t=f(x)
$$

We integrated the terms in (24) to have

$$
\begin{aligned}
& p_{o} \int_{o}^{x} \int_{o}^{u} y(t) d t d u+p_{1} \int_{o}^{x} \int_{o}^{u} t y^{\prime}(t) d t d u+p_{2} \int_{o}^{x} \int_{o}^{u} t^{2} y^{\prime \prime}(t) d t d u \\
& +\int_{o}^{x} \int_{o}^{u} \int_{a}^{b} k(z, t) y(t) d t d u d z=\int_{o}^{x} \int_{o}^{u} f(z) d t d u
\end{aligned}
$$

We simplified each term of (25) to have

$$
\begin{aligned}
& p_{o} \int_{o}^{x} \int_{o}^{u} y(t) d t d u=p_{o} \int_{o}^{x} \int_{o}^{u} \sum_{r=o}^{N} a_{r} r^{r} d t=p_{o} \int_{o}^{x} \sum_{r=o}^{N} a_{r} \frac{u^{r+1}}{r+1}=p_{o} \sum_{r=o}^{N} a_{r} \frac{u^{r+1}}{(r+1)(r+2)} \\
& p_{1} \int_{0}^{z} \int_{0}^{u} t y^{\prime}(t) d t d u=p_{1} \sum_{r=o}^{N} a_{r} \frac{x^{r+2}}{(r+1)(r+2)} \\
& p_{2} \iint t^{2} y^{\prime \prime}(t) d t d u=p_{2} \sum_{r=o}^{N} \frac{\left(r^{2}-r\right)}{(r+1)(r+2)} a_{r} x^{r+2}
\end{aligned}
$$

We let

$\int_{o}^{x} \int_{o}^{u} \int_{a}^{b} k(z, t) \sum_{r=o}^{N} a_{r} t^{r} d t d u d z=G(a, x)$ 
And

$\int_{o}^{x} \int_{o}^{u} f(z) d z d u=f(x)$

We substituted (26) - (30) into (25), we have

$p_{o} \sum_{r=o}^{N} a_{r} \frac{U^{r+2}}{(r+1)(r+2)}+p_{1} \sum_{r=o}^{N} a_{r} \frac{x^{r+2}}{(r+1)(r+2)}+p_{2} \sum_{r=o}^{N} \frac{\left(r^{2}-r\right)}{(r+1)(r+2)} a_{r} x^{r+2}+G(a, x)=F(x)$

Thus, from (30) we have

$$
\begin{aligned}
& p_{o}\left[\frac{x^{2}}{2} a_{o}+\frac{x^{3}}{6} a_{1}+\frac{x^{4}}{12} a_{2}+\frac{x^{5}}{20} a_{3}+\ldots \ldots . .+\frac{x^{N+2}}{(N+1)(N+2)} a_{N}\right] \\
& +p_{2}\left[\frac{x^{4}}{6} a_{2}+\frac{3 x^{5}}{10} a_{3}+\frac{2}{5} a_{4}+\ldots . .+\frac{\left(N^{2}-N\right) x^{N+2}}{(N+1)(N+2)} a_{N}\right]
\end{aligned}
$$

$+G(a, x)=F(x)$

Further simplification of (31) gives

$$
\begin{aligned}
& p_{o} \frac{x^{2}{ }_{k}}{2} a_{o}+\left(p_{o}+p_{1}\right) \frac{x^{3}{ }_{k}}{6} a_{1}+\frac{5}{12}\left(p_{o}+p_{1}+p_{2}\right) x^{4}{ }_{k} a_{2}+\left(p_{o}+p_{1}+p_{2}\right) \frac{x^{5}{ }_{k}}{2} a_{3} \\
& +\quad \ldots \ldots \ldots . .+\left(p_{o}+p_{1}+p_{2}\right) \frac{\left(N^{2}-N\right) x^{N+2}}{(N+1)(N+2)} a_{N}+G(a, x)=F(x)
\end{aligned}
$$

(32)

Hence, we collocated (32) at the point $x=x_{k}$ to have

$$
\begin{aligned}
& p_{o} \frac{x_{k}^{2}}{2} a_{o}+\left(p_{o}+p_{1}\right) \frac{x_{k}^{3}}{6} a_{1}+\frac{5}{12}\left(p_{o}+p_{1}+p_{2}\right) x_{k}^{4} a_{2}+\left(p_{o}+p_{1}+p_{2}\right) \frac{x_{k}^{5}}{2} a_{3}+ \\
& \ldots \ldots . .+\left(p_{o}+p_{1}+p_{2}\right) \frac{\left(N^{2}-N\right) x_{k}^{N+2}}{(N+1)(N+2)} a_{N}+G\left(a, x_{k}\right)=F\left(x_{k}\right)
\end{aligned}
$$

Where $x_{k}=a+\frac{(b-a) k}{N+1}, k=1,2,3, \ldots \ldots . \mathrm{N}+1$.

Thus, (33) is put in matrix form as described in (23), we have

$$
\left[\begin{array}{lllll}
A_{11} & A_{12} & A_{13} & \ldots & A_{1 N} \\
A_{21} & A_{22} & A_{23} \ldots & A_{2 N} \\
A_{31} & A_{32} & A_{33} & \ldots & A_{3 N} \\
: & : & : & : \\
: & : & : & & : \\
A_{N 1} & A_{N 2} & A_{N 3} \ldots & A_{N N}
\end{array}\right], X=\left[\begin{array}{l}
a_{o} \\
a_{1} \\
a_{2} \\
: \\
: \\
a_{N}
\end{array}\right] \quad \text { and } \quad b=\left[\begin{array}{l}
F\left(x_{1}\right) \\
F\left(x_{2}\right) \\
F\left(x_{3}\right) \\
: \\
: \\
F\left(x_{N+1}\right)
\end{array}\right]
$$

Where,

$$
\begin{aligned}
& A_{11}=\frac{p_{0}}{2} x_{1}^{2}+G\left(x_{1}\right), \\
& A_{12}=\left(p_{0}+p_{1}\right) x_{1}^{3}+G\left(x_{1}\right) \\
& A_{13}=\frac{5}{12}\left(p_{0}+p_{1}+p_{2}\right) x_{1}{ }^{4}+G\left(x_{1}\right)
\end{aligned}
$$




$$
\begin{aligned}
& A_{1 N}=\frac{\left(p_{0}+p_{1}+p_{2}\right)\left(N^{2}+1\right)}{(N+1)(N+2)} x_{1}^{N+1}+G\left(x_{1}\right) \\
& A_{21}=\frac{p_{0}}{2} x_{2}^{2}+G\left(x_{2}\right) \\
& A_{22}=\left(P_{0}+p_{1}\right) x_{2}^{3}+G\left(x_{2}\right) \\
& A_{23}=\frac{5}{12}\left(p_{0}+p_{1}+p_{2}\right) x^{4}{ }_{2}+G\left(x_{2}\right) \\
& A_{2 N}=\frac{\left(P_{0}+P_{1}+p_{2}\right)\left(N^{2}+1\right)}{(N+1)(N+2)} x_{2}^{\mathrm{N}+1}+G\left(x_{2}\right) \\
& A_{31}=\frac{p_{o}}{2} x \frac{2}{3}+G\left(x_{3}\right) \\
& A_{32}=\left(p_{o}+p_{1}\right) x \frac{3}{3}+G\left(x_{3}\right) \\
& A_{33}=\frac{5}{12}\left(p_{o}+p_{1}+p_{2}\right) x \frac{4}{3}+G\left(x_{3}\right) \\
& A_{3 \mathrm{~N}}=\frac{\left(p_{o}+p_{1}+p_{2}\right)\left(N^{2+1}\right.}{(N+1)(N+2)} x_{3}^{\mathrm{N}+1}+G\left(x_{3}\right) \\
& A_{N 1}=\frac{p_{o}}{2} x_{N+1}^{2}+G\left(x_{N}+1\right) \\
& A_{N 2}=\left(p_{o}+p_{1}\right) x_{R}^{3}+G\left(x_{N}+1\right) \\
& A_{N 3}=\frac{5}{12}\left(p_{o}+p_{1}+p_{2}\right) x_{N+1}^{4}+G\left(x_{N+1}\right) \\
& A_{N N}=\frac{\left(p_{o}+p_{1}+p_{2}\right)\left(N^{2}+1\right)}{(N+1)(N+2)} x_{N+1}^{N+1}+G\left(x_{N+1}\right)
\end{aligned}
$$

Remark:

The matrix is solved by Gaussian Elimination method to obtain the unknown constants $a_{i}(i \geq 0)$ which are then substituted into the approximate solution (6)

\subsubsection{Perturbed Integral Collocation Method}

An attempt to improve the accuracy of the standard integral collocation approximation $y_{N}(x)$ in section (2.1.1) is the focus of this section.

In this method, the approximate solution (6) is substituted into a slightly perturbed equation (5) to get

$$
\begin{aligned}
& \iiint \ldots . . n \ldots \ldots . . \sum_{i=o}^{n}\left(p_{i} x^{i} \frac{d^{i}}{d x^{i}}\right) y_{N}(x) d x= \\
& \iiint \ldots \ldots . \ldots \ldots . . \int\left[f(x)-\int_{a}^{b} k(x, t) d t\right] d x+H_{N}(x)
\end{aligned}
$$


Together with the condition

$L * y_{N}\left(x_{r k}\right)=\alpha_{k}, k=1(1) n$

And where,

$$
H_{N}(x)=\sum_{i=o}^{N} \tau_{r} T_{r}(x)
$$

Substituting (36) into (38), we obtain

$$
\begin{aligned}
& \iiint \ldots \ldots n \ldots \ldots . . \int \sum_{i=o}^{n}\left(p_{i} x^{i} \frac{d^{i}}{d x^{i}}\right) y_{N}(x) d x \\
& =\iiint \ldots . . n \ldots . . . . \int\left[f(x)-\int_{a}^{b} k(x, t) y(t) d t\right] d x+\tau_{1} T_{1}(x)+\ldots . .+\tau_{2} T_{N}(x)
\end{aligned}
$$

Thus, (37) is collocated at the point $x=x_{k}$, we have

$$
\begin{aligned}
& \iiint \ldots . . n \ldots \ldots . . \int \sum_{i=o}^{n}\left(p_{i} x_{k}{ }^{i} \frac{d^{i}}{d x^{i}}\right) y_{N}\left(x_{k}\right) d x \\
& =\iiint \ldots . . n \ldots \ldots . . . \int\left[f\left(x_{k}\right)-\int_{a}^{b} k\left(x_{k}, t\right) y(t) d t\right] d x+\tau_{1} T_{1}\left(x_{k}\right)+\ldots . .+\tau_{2} T_{N}\left(x_{k}\right)
\end{aligned}
$$

Where,

$$
x_{k}=a+\frac{(b-a) k}{N+1} \quad, k=1,2,3, \ldots \ldots . . \mathrm{N}+\mathrm{n}+1
$$

Remarks

The conditions are taken care after the evaluation of the integral in (38). Altogether, we obtained $(\mathrm{N}+\mathrm{n}+1)$ algebraic systems of linear equations in $(\mathrm{N}+\mathrm{n}+1)$ unknown constants $\left(a_{i}(i \geq o) \tau_{1} \tau_{2 \ldots \ldots .} \tau_{N}\right)$. These linear equation are then solved by Gaussian Elimination method to obtain $(\mathrm{N}+\mathrm{n}+1)$ unknown constants which are substituted into (6) to obtain the approximation solution for the value of $\mathrm{N}$.

To demonstrate this method further, we consider case $n=1$ in (1). After all simplification as in solution (2.1.1), we slightly perturbed (20).to obtain

$$
\sum_{r=0}^{N}\left(p_{0}+p_{1}\right) a_{r} \frac{x^{r+1}}{r+1}+G(a, x)=F(x)+\tau_{1} T_{1}(x)+\ldots \ldots .+\tau_{N} T_{N}(x)
$$

Where $G(a, x)$ and $F(x)$ are as defined in (6).

Thus, we have

$$
\begin{gathered}
P_{0} a_{0} x+\left(P_{0}+P_{1}\right) a_{1} \frac{x^{2}}{2}+\left(P_{0}+2 P_{1}\right) a_{2} \frac{x^{3}}{3}+\left(P_{0}+3 P_{1}\right) a_{3} \frac{x^{4}}{4}+\left(P_{0}+4 P_{1}\right) a_{4} \frac{x^{5}}{5}+\ldots \ldots+ \\
\left(p_{o}+N p_{1}\right) a_{N} \frac{x_{k}^{N+1}}{N+1}+G\left(a, x_{k}\right)=F\left(x_{k}\right)+\tau_{1} T_{1}\left(x_{k}\right)+\ldots \ldots \ldots . .+\tau_{N} T_{N}\left(x_{k}\right)
\end{gathered}
$$

Where

$$
x_{k}=a+\frac{(b-a) k}{N+n+1,} \quad k=1,2,3, \ldots \ldots \ldots . . \mathrm{N}+n
$$

Thus (41) is put in matrix form described in (23) where

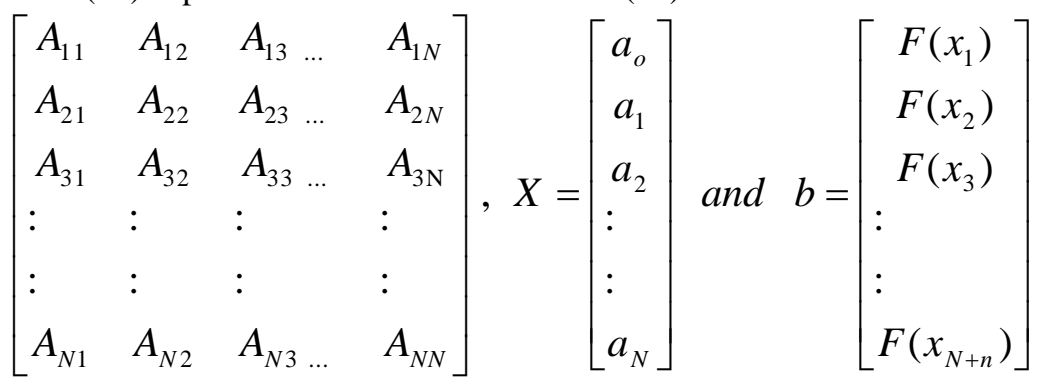

Where, 


$$
\begin{aligned}
& A_{11}=p_{o} x_{1}+G\left(x_{1}\right), \\
& A_{12}=\left(p_{o}+p_{1}\right) \frac{x_{1}^{2}}{2}+G\left(x_{1}\right) \\
& A_{13}=\left(p_{o}+2 p_{1}\right) \frac{x_{1}^{3}}{3}+G\left(x_{1}\right) \\
& A_{1 N}=\left(p_{o}+N p_{1} \frac{x_{1}^{N+1}}{N+1}+G\left(x_{1}\right)-\left(C_{o}^{(1)}+C_{1}^{(1)} x_{1}\right)\right. \\
& A_{21}=p_{o} x_{2}+G\left(x_{2}\right) \\
& A_{22}=\left(p_{o}+p_{1}\right) \frac{x_{2}^{2}}{2}+G\left(x_{2}\right) \\
& A_{23}=\left(p_{o}+2 p_{1}\right) \frac{x_{2}^{3}}{3}+G\left(x_{2}\right) \\
& A_{31}=p_{o} x_{3}+G\left(x_{3}\right) \\
& A_{32}=\left(p_{o}+p_{1}\right) \frac{x_{3}^{2}}{2}+G\left(x_{3}\right) \\
& A_{33}=\left(p_{o}+2 p_{1}\right) \frac{x_{3}^{3}}{3}+G\left(x_{3}\right) \\
& A_{3 \mathrm{~N}}=\left(p_{o}+N p_{1}\right) \frac{x_{3}^{\mathrm{N}+1}}{N+1}+G\left(x_{3}\right)-\left(C_{o}^{(1)}\right)-\left(C_{o}^{(1)} x_{3}\right) \\
& A_{N 1}=p_{o} x_{N}+G\left(x_{N}\right) \\
& A_{N 2}=\left(p_{o}+p_{1}\right) \frac{x_{N}^{2}}{2}+G\left(x_{N}\right) \\
& A_{N 3}=\left(p_{o}+2 p_{1}\right) \frac{x_{N}^{3}}{3}+G\left(x_{N}\right) \\
& A_{N N}=\left(p_{o}+N p_{1}\right) \frac{x_{N}^{N+1}}{N+1}+G\left(x_{N}\right)-\left(C_{o}^{(1)}\right)+\left(C_{1}^{(1)} x_{N}\right) \\
& A_{N+n, 1}=P_{0} x_{R}+G\left(x_{N+n}\right) \\
& A_{N+n, 2}=\left(P_{0}+P_{1}\right) \frac{x_{R}^{2}}{2}+G\left(x_{N+n}\right) \\
& A_{N+n, 3}=\left(P_{0}+2 P_{1}\right) \frac{x_{\mathrm{R}}^{3}}{3}+G\left(x_{N+n}\right) \\
& A_{N+n, N}=\left(P_{0}+N P_{1}\right) \frac{x_{N+n}^{N+1}}{N+1}+G\left(x_{N+n}\right)-\left(C_{0}^{(1)}\right)+\left(C_{1}^{(1)} x_{N+n}\right)
\end{aligned}
$$

Remark:

The matrix is solved by Gaussian Elimination method to obtain the unknown constants $\left(a_{i}(i \geq 0) \tau_{1}, \tau_{2}, \ldots ., \tau_{N}\right)$ which are then substituted into the approximate solution (6) 


$$
\begin{aligned}
& p_{o} \frac{x^{2}}{2} a_{o}+\left(p_{o}+p_{1}\right) \frac{x^{3}}{2} a_{1}+\frac{5}{12}\left(p_{o}+p_{1}+p_{2}\right) x^{4} a_{2}+\left(p_{o}+p_{1}+p_{2}\right) \frac{x^{5}}{2} a_{3}+\ldots \ldots . . \\
& +\left(p_{o}+p_{1}+p_{2}\right) \frac{\left(N^{2}+1\right) x^{N+1}}{(N+1)(N+2)} a_{N}+G(a, x)=F(x)+\tau_{2} T_{N}(x)+\ldots \ldots . \tau_{N} T_{N}(x)
\end{aligned}
$$

Hence, we collocated (43) at the point $x=x_{k}$ to have

$$
\begin{aligned}
& P_{0} \frac{x^{2}}{2} a_{0}+\left(P_{0}+P_{1}\right) \frac{x_{k}^{3}}{6} a_{1}+\frac{5}{12}\left(P_{0}+P_{1}+P_{2}\right) x_{k}^{4} a_{2}+\left(P_{0}+P_{1}+P_{2}\right) \frac{x_{k}^{5}}{2} a_{3}+\ldots . . . \\
& +\left(P_{0}+P_{1}+P_{2}\right) \frac{\left(N^{2}+1\right) x_{k}^{N+2}}{(N+1)(N+2)} a_{N}+G\left(a, x_{k}\right)=F\left(x_{k}\right)+\tau_{1} T_{1}\left(x_{k}\right)+\ldots . .+\tau_{N} T_{N}\left(x_{k}\right)
\end{aligned}
$$

Where $x_{k}=a+\frac{(b-a) k}{N+3}, \quad k=1,2,3, \ldots, \mathrm{N}+2$. Thus, (44) is put in matrix form as described in (23), where

$\left[\begin{array}{lllll}A_{11} & A_{12} & A_{13} \ldots & A_{1 N} \\ A_{21} & A_{22} & A_{23} \ldots & A_{2 N} \\ A_{31} & A_{32} & A_{33} & \ldots & A_{3 N} \\ : & : & : & : \\ : & : & : & & : \\ A_{N 1} & A_{N 2} & A_{N 3} \ldots & A_{N N}\end{array}\right], X=\left[\begin{array}{l}a_{o} \\ a_{1} \\ a_{2} \\ : \\ : \\ a_{N}\end{array}\right]$ and $b=\left[\begin{array}{l}F\left(x_{1}\right) \\ F\left(x_{2}\right) \\ F\left(x_{3}\right) \\ : \\ : \\ F\left(x_{N+2}\right)\end{array}\right]$

and

$$
\begin{aligned}
& A_{11}=\frac{p_{o}}{2} x_{1}^{2}+G\left(x_{1}\right), \\
& A_{12}=\left(p_{o}+p_{1}\right) \frac{x_{1}^{2}}{6} G\left(x_{1}\right) \\
& A_{13}=\frac{5}{12}\left(p_{o}+p_{1}+p_{2}\right) x_{1}^{4}+G\left(x_{1}\right) \\
& A_{14}=\left(p_{o}+p_{1}+p_{2}\right) \frac{x_{1}^{5}}{2}+G\left(x_{1}\right) \\
& A_{1 N}=\frac{\left(p_{o}+p_{1}+p_{2}\right)\left(N^{2}+1\right)}{(N+1)(N+2)} x_{1}^{N+1}+G\left(x_{1}\right)-\left(C_{o}^{(1)}+C_{1}^{(1)} x_{1}\right)-\left(C_{o}^{(2)}+C_{1}^{(2)} x_{1}+C_{2}^{(2)} x_{1}^{2}\right) \\
& A_{21}=\frac{p_{o}}{2} x_{2}^{2}+G\left(x_{1}\right) \\
& A_{22}=\left(p_{o}+p_{1}\right) \frac{x_{2}^{3}}{6}+G\left(x_{1}\right) \\
& A_{23}=\frac{5}{12}\left(p_{o}+p_{1}+p_{2}\right) x_{2}^{4}+G\left(x_{1}\right) \\
& A_{24}=\left(p_{o}+p_{1}+p_{2}\right) \frac{x_{2}^{5}}{2}+G\left(x_{1}\right) \\
& A_{2 N}=\frac{\left(p_{o}+p_{1}+p_{2}\right)\left(N^{2}+1\right)}{(N+1)(N+2)} x_{2}^{N+2}+G\left(x_{2}\right)-\left(C_{o}^{(1)}+C_{1}^{(1)} x_{2}\right)-\left(C_{o}^{(2)}+C_{1}^{(2)} x_{2}+C_{2}^{(2)} x_{2}^{2}\right) \\
& A_{31}=\frac{p_{o}}{2} x_{3}^{2}+G\left(x_{3}\right)
\end{aligned}
$$




$$
\begin{aligned}
& A_{32}=\left(p_{o}+p_{1}\right) \frac{x_{3}^{3}}{6}+G\left(x_{3}\right) \\
& A_{33}=\frac{5}{12}\left(p_{o}+p_{1}+p_{2}\right) x_{3}^{4}+G\left(x_{3}\right) \\
& A_{34}=\left(p_{o}+p_{1}+p_{2}\right) \frac{x_{3}^{5}}{2}+G\left(x_{3}\right) \\
& A_{3 N}=\frac{\left(p_{o}+p_{1}+p_{2}\right)\left(N^{2}+1\right)}{(N+1)(N+2)} x_{3}^{N+2}+G\left(x_{3}\right)-\left(C_{o}^{(1)}+C_{1}^{(1)} x_{3}\right)-\left(C_{o}^{(2)}+C_{1}^{(2)} x_{3}+C_{2}^{(2)} x_{3}^{2}\right) \\
& A_{N 1}=\frac{p_{o}}{2} x_{N}^{2}+G\left(x_{N}\right) \\
& A_{N 2}=\left(p_{o}+p_{1}+\right) \frac{x_{2}^{3}}{6}+G\left(x_{N}\right) \\
& A_{\mathrm{N} 3}=\frac{5}{12}\left(p_{o}+p_{1}+p_{2}\right) x_{\mathrm{N}}^{4}+G\left(x_{\mathrm{N}}\right) \\
& A_{N 4}=A_{N 4} \\
& A_{N N}=\frac{\left(p_{o}+p_{1}+p_{2}\right)\left(N^{2}+1\right)}{(N+1)(N+2)} x_{N}^{N+2}+G\left(x_{N}\right)-\left(C_{o}^{(1)}+C_{1}^{(1)} x_{N}\right)-\left(C_{o}^{(2)}+C_{1}^{(2)} x_{N}+C_{2}^{(2)} x_{N}^{2}\right) \\
& A_{\mathrm{N}+2,1}=\frac{p_{o}}{2} x_{\mathrm{N}+2}^{2}+G\left(x_{N+2}\right) \\
& A_{N+2, N}=\left(p_{o}+p_{1}+p_{2}\right) \frac{x_{N+2}^{5}}{2}+G\left(x_{N+2}\right) \\
& A_{\mathrm{N}+2,2}=\frac{5}{12}\left(p_{o}+p_{1}+p_{2}\right) x_{\mathrm{N}+2}^{4}+G\left(x_{\mathrm{N}+2}\right) \\
& :
\end{aligned}
$$

Remarks

The above matrix is solved by Gaussian Elimination method to obtain the unknown constants $a_{i}(i \geq o)$ which are then substituted into the approximate solution (6)

\section{Numerical Demonstration.}

In this section, we have demonstrated the method discussed here on four examples, two first and two second orders integro differential equations. We have defined error as

$$
\text { Error }=\text { Error }=\left|y(x)-y_{N}(x)\right|, \quad a \leq x \leq b
$$

\section{Example 1:}

We consider the following first order integro differential equation of the form

$$
y^{\prime}(x)-\int_{0}^{1} 3 x t y(t) d t=3 e^{3 x}-\frac{1}{3}\left(2 x^{3}+1\right)
$$

With condition given as $y(0)=1$ and the exact solution as $y(x)=e^{3 \mathrm{x}}$

\section{Example 2:}

$$
y^{\prime}(x)-y(x)-\int_{0}^{1} \operatorname{Sin}(4 \pi x-2 \pi t) y(t) d t=-\operatorname{Cos}(2 \pi x)-2 \pi \operatorname{Sin} 2 \pi x-\frac{1}{2} \operatorname{Sin}(4 \pi x)
$$


With the condition $y(0)=1$ and the exact solution is given as $y(x)=\operatorname{Cos}(2 \pi x)$

Example 3:

$y^{\prime \prime}(x)+\int_{0}^{\frac{\pi}{2}} x t y(t) d t=x-\operatorname{Sin} x, \quad 0 \leq x \leq \frac{\pi}{2}$

With the conditions given as

$y(0)=0$ and $y^{\prime}(0)=1$

The exact solution is given as $y(x)=\operatorname{Sin} x$

\section{Example 4:}

$y^{\prime \prime}(x)+4 x y(x)+2 \int_{0}^{1} \frac{t^{2}+1}{x^{2}+1} y(t) d t=-\frac{8 x^{4}}{\left(x^{3}+1\right)^{3}}, \quad 0 \leq x \leq 1$

With the conditions $y(0)=1$ and $y^{\prime}(0)=\frac{1}{2}$ The exact solution is $y(x)=\left(x^{2}+1\right)^{-1}$

IV. Tables of Results

Table 1: Errors obtained from example 1 for different values of $\mathbf{N}$

\begin{tabular}{|c|c|c|c|c|c|c|}
\hline $\mathbf{X}$ & $\begin{array}{l}\text { HAM[6] } \\
\text { N=10 }\end{array}$ & $\begin{array}{l}\text { HAM[6] } \\
\text { N=20 }\end{array}$ & $\begin{array}{l}\text { Standard } \\
\text { integral } \\
\text { collocation } \\
\text { method } \\
\mathrm{N}=10\end{array}$ & $\begin{array}{l}\text { Standard } \\
\text { integral } \\
\text { collocation } \\
\text { method } \\
\mathrm{N}=20\end{array}$ & $\begin{array}{l}\text { Perturbed } \\
\text { integral } \\
\text { collocation } \\
\text { method } \\
\mathrm{N}=10\end{array}$ & $\begin{array}{l}\text { Perturbed } \\
\text { integral } \\
\text { collocation } \\
\text { method } \\
\mathrm{N}=20\end{array}$ \\
\hline 0 & 0 & 0 & 0 & 0 & 0 & 0 \\
\hline 0.2 & $9.50054 \mathrm{E}-9$ & $5.25769 \mathrm{E}-9$ & $6.12604 \mathrm{E}-6$ & $3.14032 \mathrm{E}-9$ & $8.05735 \mathrm{E}-7$ & 4. $32105 \mathrm{E}-10$ \\
\hline 0.4 & $3.82421 \mathrm{E}-4$ & 2.10307E-8 & 8.03261E-5 & $1.86232 \mathrm{E}-8$ & 7. $32561 \mathrm{E}-5$ & 2.00532E-9 \\
\hline 0.6 & $8.60448 E-4$ & 4.73192E-8 & 1.61293E-4 & $3.17031 \mathrm{E}-8$ & 6.00532E-5 & 1.27832E-8 \\
\hline 0.8 & $1.52968 \mathrm{E}-5$ & 8.41230E-8 & $9.04215 E-5$ & 2.14652E-8 & 7. $32406 \mathrm{E}-4$ & 2.57342E-9 \\
\hline 1 & 2.30013E-3 & 1.33442E-7 & $1.76112 \mathrm{E}-3$ & $2.01142 \mathrm{E}-8$ & $1.25672 \mathrm{E}-4$ & $1.11456 \mathrm{E}-8$ \\
\hline
\end{tabular}

Table 2: Errors obtained from example 2 for different values of $\mathbf{N}$

\begin{tabular}{|c|c|c|c|c|c|c|}
\hline $\mathbf{X}$ & $\begin{array}{l}\text { HAM[6] } \\
\text { N=10 }\end{array}$ & $\begin{array}{l}\text { HAM[6] } \\
\mathrm{N}=20\end{array}$ & $\begin{array}{l}\text { Standard } \\
\text { integral } \\
\text { collocation } \\
\text { method } \\
\mathrm{N}=10\end{array}$ & $\begin{array}{l}\text { Standard } \\
\text { integral } \\
\text { collocation } \\
\text { method } \\
\mathrm{N}=20\end{array}$ & $\begin{array}{l}\text { Perturbed } \\
\text { integral } \\
\text { collocation } \\
\text { method } \\
\mathrm{N}=10\end{array}$ & $\begin{array}{l}\text { Perturbed } \\
\text { integral } \\
\text { collocation } \\
\text { method } \\
\mathrm{N}=20\end{array}$ \\
\hline 0. & $\mathbf{0}$ & $\mathbf{0}$ & 0 & $\mathbf{0}$ & $\mathbf{0}$ & 0 \\
\hline 0.2 & $2.5701 \mathrm{E}-4$ & 4.2117E-6 & 2.1326E-4 & 2. $3204 \mathrm{E}-6$ & $2.0756 \mathrm{E}-4$ & $7.5461 \mathrm{E}-7$ \\
\hline 0.4 & 4.8001E-4 & $5.8797 \mathrm{E}-9$ & 4.1003E-4 & $7.1325 \mathrm{E}-9$ & $3.9321 \mathrm{E}-4$ & 8.5323E-10 \\
\hline 0.6 & $9.7723 \mathrm{E}-3$ & $4.2112 \mathrm{E}-8$ & $2.9412 \mathrm{E}-5$ & $9.7214 \mathrm{E}-9$ & 5. $3451 \mathrm{E}-5$ & $5.0176 \mathrm{E}-9$ \\
\hline 0.8 & $9.7723 \mathrm{E}-3$ & 6.4123E-8 & $1.7325 \mathrm{E}-3$ & 5.0321E-8 & $8.1463 \mathrm{E}-4$ & 3. $3742 \mathrm{E}-8$ \\
\hline 1 & $1.3021 \mathrm{E}-3$ & 1.2314E-8 & 1.0027E-3 & 1.1327E-8 & 9. $3241 \mathrm{E}-4$ & 1.1343E-9 \\
\hline
\end{tabular}

Table 3: Maximum errors obtained for example 3

\begin{tabular}{|c|c|c|c|c|}
\hline $\mathrm{N}^{\text {Method }}$ & $\begin{array}{ll}\text { Chebyshev } & \text { Polynomial } \\
\text { Approximation } & \\
{[10]} & \\
& \end{array}$ & $\begin{array}{l}\text { Chebyshev Polynomial } \\
\text { Approximation } \\
{[10]}\end{array}$ & $\begin{array}{ll}\text { Standard } & \text { Integral } \\
\text { Collocation Method }\end{array}$ & $\begin{array}{l}\text { Perturbed Integral } \\
\text { Collocation Method }\end{array}$ \\
\hline 6 & $1.32 \mathrm{E}-5$ & $1.75 \mathrm{E}-4$ & $1.16 \mathrm{E}-4$ & $2.24 \mathrm{E}-6$ \\
\hline 8 & $1.17 \mathrm{E}-9$ & $3.08 \mathrm{E}-8$ & $1.03 \mathrm{E}-9$ & $2.56 \mathrm{E}-10$ \\
\hline 10 & $1.25 \mathrm{E}-11$ & $1.35 \mathrm{E}-9$ & $2.04 \mathrm{E}-11$ & $3.16 \mathrm{E}-12$ \\
\hline
\end{tabular}


Table 4: Errors of Example 4

\begin{tabular}{|l|l|l|l|}
\hline $\mathrm{X}$ & $\begin{array}{l}\text { Wavelet } \\
\text { Galerkin }\{8\} \\
\mathrm{N}=6\end{array}$ & $\begin{array}{l}\text { Standard } \\
\text { Integral } \\
\text { Collocation Method } \\
\mathrm{N}=6\end{array}$ & $\begin{array}{l}\text { Perturbed } \\
\text { Integral } \\
\text { Collocation Method } \\
\mathrm{N}=6\end{array}$ \\
\hline 0 & 0 & 0 & 0 \\
\hline 0.125 & $2.7 \mathrm{E}-4$ & $1.3 \mathrm{E}-4$ & $8.6 \mathrm{E}-5$ \\
\hline 0.250 & $3.1 \mathrm{E}-5$ & $2.8 \mathrm{E}-5$ & $2.6 \mathrm{E}-5$ \\
\hline 0.375 & $2.6 \mathrm{E}-4$ & $7.4 \mathrm{E}-5$ & $1.7 \mathrm{E}-5$ \\
\hline 0.500 & $4.3 \mathrm{E}-4$ & $3.2 \mathrm{E}-4$ & $3.9 \mathrm{E}-6$ \\
\hline 0.625 & $5.6 \mathrm{E}-4$ & $5.1 \mathrm{E}-4$ & $6.3 \mathrm{E}-5$ \\
\hline 0.750 & $6.6 \mathrm{E}-4$ & $1.9 \mathrm{E}-5$ & $4.6 \mathrm{E}-6$ \\
\hline 0.875 & $7.2 \mathrm{E}-4$ & $6.2 \mathrm{E}-5$ & $3.5 \mathrm{E}-5$ \\
\hline 1 & 0 & 0 & 0 \\
\hline
\end{tabular}

\section{Conclusion}

This paper has employed successfully standard and perturbed integral collocation method to solve special first and second orders linear integro-differential equation.

Power series form of approximation is used as basis function, the application of standard and perturbed integral collocation methods on some problems including linear first and second orders are considered. The most important ones are the simplicity of the methods.

Furthermore, these methods yield the desired accuracy when compared the results obtain with the exact solutions.

All these advantage of the Standard and Perturbed integral collocation methods to solve first and second orders linear integro-differential equation assert that the methods are convenient, reliable and powerful tools for the classes of the problem considered.

\section{References}

[1] Aliyu, I . M. (2012) A computational error estimation of the Integral Formulation of the Tau Method for some class of ordinary differential equations. Ph. D Thesis (Unpublished), University of Ilorin, Nigeria.

[2] Asady, B. and Kajani, M.T. (2005). Direct Method for solving Integro Differential Equation using Hybrid Fourier and Block Pulse Function. Intern. J . Computer Math., 82(7), 989-995.

[3] Ayad, A. (1996). Spline Approximation for the First Order Fredhom Integral Differential Equations. University Babes Bolyai, Studia, Mathematics, 41(3), 1-8

[4] Behiri, S . H. and Hashish H. (2002). Wavelet Methods for the numerical solution of Fredholm Integro Differential Equations. Intern. J. Applied Mathematics. 11(1), 27-33

[5] Danfu, H . and Xafeng, R. C. (2001). Numerical solution of Integro Differential Equations by using Walet Operational Matrix of Integration second kind. Applied Math. Computer. 194(2), 460-466

[6] Golbadai, H.and Javidi, M. (2007). Application of He's Homotopy Perturbation Method for the nth order Integro Differential Equations. Applied Math. Computer, 190(2), 1409 - 1419

[7] Hussein, J. Omar A. and Al-Shara, S (2008). Numerical Solution of Linear Integro Differential Equations. J. Mathematics and Statistics. 4(4), $250-254$

[8] Lekestani, M. Razzaghi, M. and Dhglhan. M. (2005). Semi Orthogonal Spline Wavelet's Approximation for Fredholm Integro Differential Equations. Hindawi Publishing Corporation, Mathematical Problem in Engineering. 2, 1-12

[9] Maleknejad, K. and Mirzace, F. (2006). Numerical solution of Integro Differential Equations using Rationalized Haar Functions Method. Keynote Intern. J. Syst. Math. 35, 1735-1744

[10] Raji, M. T. (2013). Collocation Approximation for solving Special Linear and Nonlinear Integro Differential Equations. Ph.D. Thesis, (Unpublished), University of Ilorin, Ilorin, Nigeria.

[11] Taiwo, O. A. (2007). Comparison of two collocation Methods for the Solution of Integral Equations. Journal of the Mathematical Association of Nigeria. 34(2B), 289-294.

[12] Taiwo, O . A. and Ishola, C .Y. (2009). Collocation Approximation Methods for the numerical solution of Integral Equations. Intern. J. Pure and Applied Sc. 2, 20-37.

[13] Vahidi, A. R, Babolian, E, Asady, C . G. and Azimzadeh, Z (2009). Numerical solution of Fredholm Integro Differential Equations by Adomian Decomposition Method. Intern. J. of Math. Analysis, 3(36), 1769-1773. 